\title{
Multi-Sensor Health Diagnosis Using Deep Belief Network Based State Classification
}

\author{
Prasanna Tamilselvan \\ Faculty: Pingfeng Wang
}

Department of Industrial and Manufacturing Engineering

\begin{abstract}
Effective health diagnosis provides multifarious benefits such as improved safety, reliability and economical maintenance of complex engineered systems. This paper presents a novel multi-sensor health diagnosis method using Deep Belief Network (DBN) based state classification. The DBN employs a hierarchical structure with multiple stacked Restricted Boltzmann Machines and works through a layer by layer successive learning process. The proposed multi-sensor health diagnosis can be structured in three consecutive stages: first, defining health states and collecting sensory data for DBN training and testing; second, developing DBN based classification models for the diagnosis of predefined health states; third, validating DBN classification models with testing sensory dataset. The performance of proposed DBN health state classification is compared with four other existing classification methods and demonstrated with a case study.
\end{abstract}

\section{Introduction}

Research on real-time condition monitoring and diagnosis which interprets data acquired by smart sensors and distributed sensor networks, and utilizes these data streams in making critical decisions advances significantly across a wide range of applications. This provides multifarious benefits such as improved safety, improved reliability and reduced costs for the operation and maintenance of complex engineered systems. However, effective diagnosis of current health state from multidimensional sensory data is still an intricate problem and remains as a major challenge for the application of condition monitoring techniques to complex engineered systems. To tackle this challenge, a novel multisensor health diagnosis method using DBN based state classification is developed in this study. Section 2 presents the proposed methodology with a case study on aircraft wind structure health diagnosis.

learning process of the DBN classifier model can be successively accomplished in three stages, first, unsupervised learning by RBM learning rule; second, supervised learning using back-propagation method; and third, fine tuning using the conjugate gradient algorithm. The trained DBN classifier model will be validated using the misclassification errors of both training and testing data.
Section 3 summarizes the presented research and the future work.

\section{DBN Based Health State Classification for Diagnosis}

This section introduces the multi-sensor health diagnosis using DBN based state classification technique. DBN employs a multilayered architecture which consists of one visible layer and multiple hidden layers (Fig. 1). Visible layer of DBN accepts the input data and transfers the data to the hidden layers in order to complete the machine learning process [1]

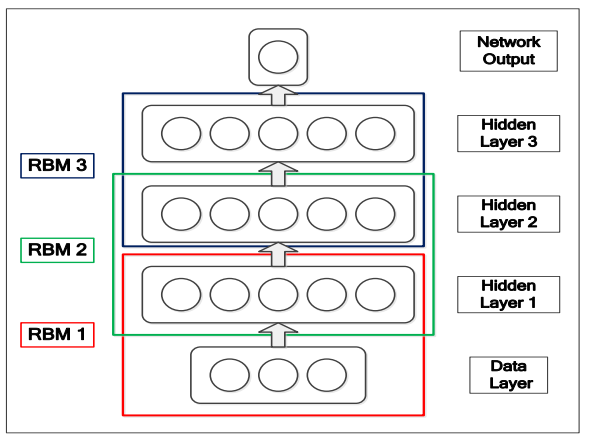

Fig. 1: Deep Belief Network Structure

The DBN structure is similar to the stacked network of the Restricted Boltzmann Machines (RBM) as shown in Fig. 1. Each RBM consists of two layers, namely visible layer and hidden layer. The process of transformation of data from visible layer to the hidden layer is finished through a sigmoid activation function based on RBM learning rule [1]. Each successive layer in the DBN follows the same transformation concept and passes the regularity throughout the DBN architecture. The overall 2.1 Procedure for DBN Health State Classification The procedure of the proposed multi-sensor health diagnosis using the DBN based state classification is shown in Table 1 . The primary step is to define the diagnosis problem and appropriate health states. Collecting data from sensors and preprocessing the data is the next part of the process. Preprocessed sensory data with known classes will be used to develop and train the DBN classifier model in steps 3 
and 4. The DBN architecture is altered based on the input patterns with different known health states. DBN classifier models will be validated using misclassification error determination process in step 5. Steps 4 and 5 will be iteratively repeated until the model reaches the minimum misclassification error. Trained DBN classifier models will be set to classify unknown sensor signals to the corresponding health states.

Table 1: Procedure for DBN Classification

\begin{tabular}{ll}
\hline Step 1: & Define the diagnosis problem and health states \\
Step 2: & $\begin{array}{l}\text { Collect and preprocess the sensory data for each } \\
\text { predefined health state }\end{array}$ \\
Step 3: & $\begin{array}{l}\text { Develop DBN classifier models } \\
\text { Step 4: }\end{array}$ \\
Train DBN classifier models using training data \\
Step 5: & $\begin{array}{l}\text { Validate the DBN classifier models } \\
\text { Perform diagnosis using trained DBN classifier } \\
\text { models }\end{array}$ \\
\hline
\end{tabular}

\subsection{Aircraft Wing Structure Health Diagnosis}

The proposed DBN classification technique is demonstrated with aircraft wing structure health diagnosis case study. One rectangular panel with two symmetric plates of the aircraft wing structure, as shown in Fig. 2, is modeled and simulated in ANSYS. The four end corners of the wing structure are fixed with bolt joint connections. The sensors are allocated in the center of the wing structure to analyze the vibrations. The variable cyclic load is applied at the center of the left side plate. Sensor network detects the aircraft wing structure degradation in three health state conditions: first, no fault condition (Health State 1, HS1), second, fault in bolt joint A (Health State 2, HS2) and third, fault in bolt joint B (Health State 3, HS3). 400 sets of random samples are generated for each health state from the aircraft wing structure simulation model considering the uncertain load condition applied. The first 200 sets of random samples are used as training data and the rest are saved as testing data. The DBN classifier model is trained by following the procedure shown in Table 1 with the training data and validated using the testing data. The trained DBN classifier model successfully classifies the training data and the testing data with high classification rates. The developed diagnosis methodology is compared with four existing approaches: Self Organizing Maps (SOM) [2]. Back propagation Neural Networks (BNN), Mahalanobis Distance (MD), and Support
Vector Machine (SVM) and the comparison results are shown in Table 2.

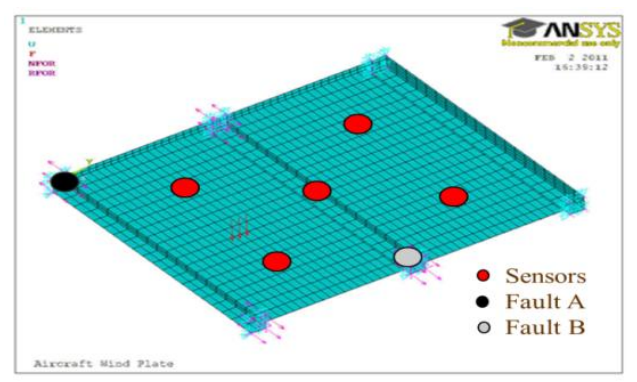

Fig. 2: Sensor Network and Fault Locations

Table 2: State Classification Results Comparison

\begin{tabular}{ccc}
\hline $\begin{array}{c}\text { Classification } \\
\text { Techniques }\end{array}$ & $\begin{array}{c}\text { Classification Rate } \\
\text { Training }(\%)\end{array}$ & $\begin{array}{c}\text { Classification Rate } \\
\text { Testing }(\%)\end{array}$ \\
\hline BNN & 86 & 85.67 \\
SOM & 79.83 & 79.17 \\
MD & 91.67 & 92.00 \\
SVM & 100 & 88.77 \\
DBN & 100 & 96.50 \\
\hline
\end{tabular}

\section{Conclusions}

This paper presented a novel multi-sensor health diagnosis method using DBN based state classification. The feasibility of the developed methodology for structure health diagnosis was demonstrated with aircraft wing structure case study and the performance was compared with four existing diagnostics methods. Case study results indicated that the developed diagnosis methodology generally produces a higher classification rate for multi-sensor health diagnosis of complex systems, compared with four existing classification methods. The DBN will be further investigated for the multi-sensor health prognosis.

\section{REFERENCES}

[1] G. E. Hinton, S. Osindero and Y. W. Teh, "A Fast Learning Algorithm for Deep Belief Nets," Neural Computation, Vol. 18, pp. 1527-1554, 2006.

[2] R. Huang, L. Xi, X. Li, C. Richard Liu, H. Qiu and J. Lee, "Residual Life Predictions for Ball Bearings Based on SelfOrganizing Map and Back Propagation Neural Network Methods," Mechanical Systems and Signal Processing, Vol. 21, pp. 193-207, 2007. 